\title{
First Report of Abnormal Spermathecae in Phlebotomus (Larroussius) longicuspis Nitzulescu, 1930 (Diptera: Psychodidae), in Tunisia
}

\author{
Jomaa Chemkhi ${ }^{1}$, Souheila Guerbouj ${ }^{1}$, Ikram Guizani ${ }^{1}$ and Afif Ben Salah ${ }^{2}$ \\ 1. Laboratoire d'Epidémiologie Moléculaire et Pathologie Expérimentale Appliquée aux Maladies infectieuses (LR11IPT04), Institut \\ Pasteur de Tunis, Université Tunis El Manar, Tunisia \\ 2. Laboratoire d’Epidémiologie Médicale, Institut Pasteur de Tunis, Université Tunis El Manar, Tunisia
}

\begin{abstract}
Phlebotomine sandflies (Diptera: Psychodidae) constitute a large group of flies, many of which have been described as vectors of leishmaniasis, a disease caused by Leishmania (Kinetoplastida: Trypanosomatidae) parasites. In Tunisia, a total of 17 species belonging to both genera Phlebotomus and Sergentomyia were described. In this work we report on an abnormality found in spermathecae of a female specimen of Phlebotomus (Larroussius) longicuspis Nitzulescu, 1930. Sandflies were collected in SidiSaad locality, in the governorate of Kairouan, central Tunisia, where zoonotic cutaneous leishmaniasis is endemic, during the June to November 2011 period, using CDC light traps. Males were washed and directly identified and females were dissected and morphologically identified using the head and the last two abdominal segments containing spermathecae and ducts. 162 flies were morphologically described. Among 33 females identified as Phlebotomus (Larroussius) longicuspis, one specimen was found abnormal and presented with three spermathecae. An additional spermathecae located in a short bifurcation on the upper extremity of one duct was found. This anomaly is for the first time described in Tunisia. Morphological abnormalities are important to report in order to avoid erroneous sandfly identification or description of new species.
\end{abstract}

Key words: Phlebotomine sand fly, spermathecae, anomaly.

\section{Introduction}

Phlebotomine sand flies (Diptera: Psychodidae: Phlebotominae) play a significant role in infectious human and animal pathologies. They constitute a large group of flies with about 900 described species which are widely distributed in tropical and temperate areas. Many sand flies are proven vectors of human disease agents including parasitic protozoa (Leishmania (L.) spp.) [1-3], bacteria (Bartonella spp.) [4, 5] and viruses (mainly Phlebovirus, Vesiculovirus and Orbivirus) [6]. In particular, 98 sand fly species are proven or suspected vectors of human leishmaniases; these include 42 Phlebotomus species in the Old

\footnotetext{
Corresponding author: Afif Ben Salah, professor, M.D., research field: epidemiology. E-mail: afif.bensalah@pasteur.rns.tn.
}

World and 56 Lutzomyia species in the New World (all: Diptera: Psychodidae) [7]. In the Old World, among the 42 Phlebotomus species, 20 are implicated in the transmission of L. infantum, 6 in L. donovani, 7 in L. major, 7 in L. tropica and 3 in the transmission of L. aethiopica [7]. Each species appears to be involved in the transmission of one Leishmania species only, except Phlebotomus sergenti and Phlebotomus alexandri which were incriminated in the transmission of both $L$. tropica and L. aethiopica and of L. donovani and L. infantum, respectively [7]. However, the 56 Lutzomyia species recorded in the New World are involved in the transmission of 15 Leishmania species and some of them are probably able to transmit more than one Leishmania species, like Lutzomyia migonei that has been found to be infected with four different parasite species [7]. 
In Tunisia, a total of 17 phlebotomine sand fly species belonging to both genera Phlebotomus (P.) and Sergentomyia (S.) were described. These genera are subdivided into six sub-genera: Sergentomyia Sergentomyia, Sergentomyia Sintonius, Sergentomyia Grassomyia, Phlebotomus Phlebotomus (P.), Phlebotomus Paraphlebotomus and Phlebotomus Larroussius (L.). The latter is constituted of five species: P. (L.) perniciosus Newstead, 1911, P. (L.) longicuspis Nitzulescu, 1930, P. (L.) langeroni Nitzulescu, 1930, P. (L.) perfiliewi Parrot, 1939, P. (L.) ariasi Tonnoir, 1921 and P. (L.) chadlii Rioux, Juminer \& Gibily, 1966; Chamkhi, 2006. Both P. (L.) perniciosus and $P$. (L.) longicuspis species are most frequently found in Tunisian semi-arid and arid bioclimate zones [8]. The species $P$. (L.) perniciosus have been described as the main vector of visceral leishmaniasis due to L. infantum, not only in Tunisia but also in neighbouring Mediterranean countries while Parrot et al. [9] were the first to propose $P$. (L.) longicuspis as a potential vector of visceral leishmaniasis. Later, this species was epidemiologically identified as the vector of $L$. infantum in arid bioclimatic zones where it is particularly abundant [10, 11]. Recently, $P$. (L.) longicuspis was demonstrated to be naturally infected with L. infantum, in Algeria [12] and with Leishmania spp. in Tunisia [13].

Phlebotomine species morphological identification is based for males, on the genitalia, while it is based mainly on specific characteristics of the spermathecae and ducts, for female specimens [3, 14]. The species $P$. (L.) perniciosus and $P$. (L.) longicuspis are closely related. Indeed, morphological identification of $P$. (L.) perniciosus and its sympatric species $P$. (L.) longicuspis are based on their genital apparatus. Until the year 1983, females of these two species, considered to be indistinguishable, were identified only by cohabitation (presence of corresponding male in the same place of capture). However, this criterion is not valid for sympatric populations. Currently, these females are distinguished according to the form and position of the dilatation on the spermathecae duct [15].

In this work we report on a rare abnormality found in the spermathecae number of a female specimen of the species Phlebotomus (Larroussius) longicuspis.

\section{Materials and Methods}

\subsection{Study Site and Sample Collection}

In order to estimate the infection rate of phlebotomine sand fly vectors with Leishmania parasites in a focus of zoonotic cutaneous leishmaniasis (ZCL), sand flies were collected in the governorate of Kairouan, central Tunisia, during the June to November 2011 period. Kairouan is an old ZCL focus where the $L$. major epidemiological life cycle has been well described [16]. Several localities were studied in this region but this work will focus only on the locality of Sidi Saad which belongs to an arid bioclimatic zone and is situated in between two rocky hills, $3.5 \mathrm{~km}$ away from the dam of Sidi Saad (Fig. 1). CDC light traps were placed overnight, in different biotopes (inside habitations, outdoors and within animal shelters).

\subsection{Sand fly Identification}

Specimens were collected from the CDC traps with a manual capture tube. Males were washed, mounted and directly identified under a microscope. Females which were alive at collection time were immediately dissected in a drop of saline water. The head and genitalia were removed and mounted in a drop of Marc-André for morphological identification based on the keys of Lewis [17] and Léger et al. [15]. Further, the mid-gut of females suspected to be infected with Leishmania promastigotes were examined by light microscopy in a drop of sterile saline (0.9\%o). Dead sand flies were stored in liquid nitrogen and taken to the laboratory for later identification and Leishmania infection study by molecular tools. 

Nitzulescu, 1930 (Diptera: Psychodidae), in Tunisia
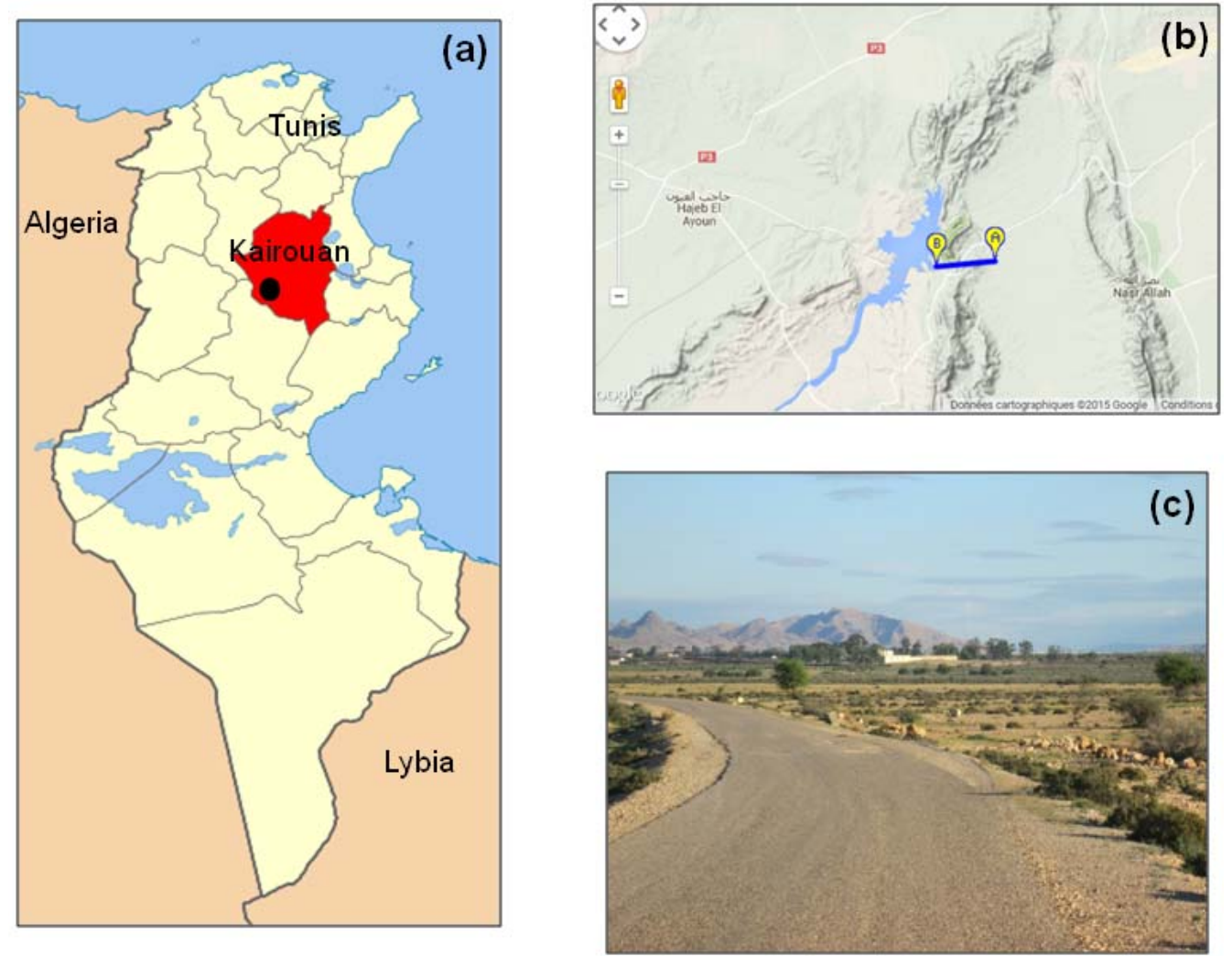

Fig. 1 Localization of the study site within the governorate of Kairouan in Tunisia.

(a) Map of Tunisia showing the Kairouan region, central Tunisia. The locality of Sidi Saad is indicated with a filled black dot. (b) The locality of Sidi Saad (marked with "A") where the study was realized, which is situated in between tow rocky hills at $3.5 \mathrm{~km}$ away from the dam of Sidi Saad (marked with “B”), as shown by Google Map. (c) Geographical localization of an example of station. (Geographical reference: N: 35²1mn 735s; E: 009 43mn 567s; Alt: 248m).

\section{Results}

A total of 162 sand flies were captured in the locality of Sidi Saad in Kairouan region, within the animal shelters biotope. Upon dissection and morphological inspection, the species were identified; both Phlebotomus and Sergentomyia genera were found. Three species (P. (P.) papatasi Scopoli, 1786, P. (L.) perniciosus Newstead, 1911 and P. (L.) longicuspis Nitzulescu, 1930) belonging to Phlebotomus and Larroussius subgenera were found within the Phlebotomus genus, while only one species (S. Grassomyia dreyfussi Parrot, 1933) was found within the Sergentomyia genus (Table 1). P. (P.) papatasi (90/162) and P. (L.) longicuspis (64/162) were the predominant species (Table 1).
Out of the 33 females identified as $P$. (L.) longicuspis, one specimen presented with an abnormal number of spermathecae. Morphological criteria that allowed identifying the sand flies as $P$. (L.) longicuspis species was the presence of a sub-terminal lateral bulb on the base of the ducts and the presence of a segmented spermathecae body, with a long neck and terminal process (Fig. 2). While females of phlebotomine sand flies have naturally two spermathecae (Fig. 2), in this abnormal specimen, one supernumerary spermathecae was found (Fig. 3). The additional spermathecae was found to be located in a short bifurcation on the upper extremity of one duct. Its body is smaller than the other pair of spermathecae with less rings and a neck without a terminal process (Fig. 3). 

Nitzulescu, 1930 (Diptera: Psychodidae), in Tunisia

Table 1 Phlebotomine sand fly species identified in the studied locality of Sidi Saad, in Kairouan region, central Tunisia.

\begin{tabular}{llllll}
\hline Genus & Sub-genus & Species & Males & Females & Total \\
\hline \multirow{3}{*}{ Phlebotomus } & Phlebotomus & papatasi & 5 & 85 & 90 \\
& Larroussius & perniciosus & 2 & 5 & 7 \\
Sergentomiya & Grassomyia & longicuspis & 31 & 33 & 64 \\
\hline
\end{tabular}

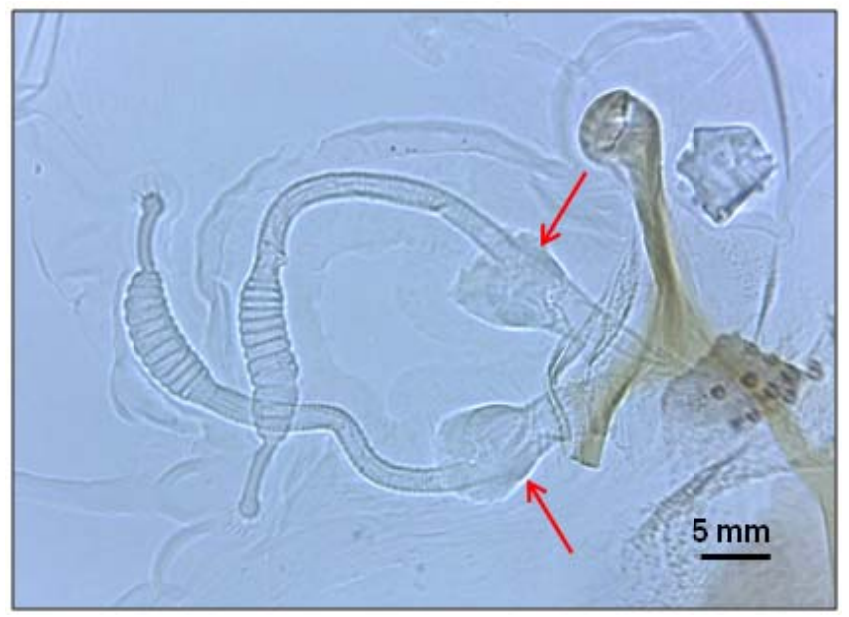

Fig. 2 Normal spermathecae of Phlebotomus (Larroussius) longicuspis species.

The ducts distal portions, characterized with lateral bulb are indicated with arrows.
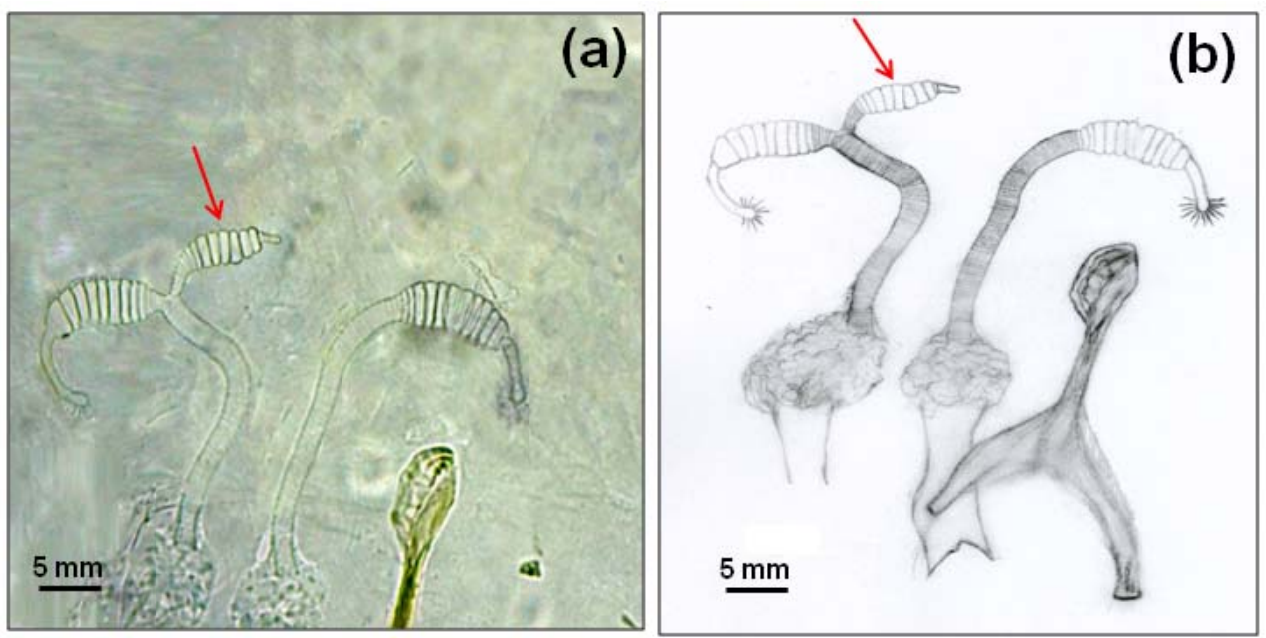

Fig. 3 Abnormal spermathecae of Phlebotomus (Larroussius) longicuspis.

(a) photographed and (b) hand drawn. The arrow indicates the abnormal spermathecae.

\section{Discussion}

Description of morphological anomalies of phlebotomine sand flies have been recorded in both the New World and the Old World. The majority of these anomalies are associated with the genitalia, the number of cibarial teeth, wing venation, the size of the palps, and other less significant features.

Sand fly males are found most frequently affected than females with supernumerary style spines being the most common anomaly described. Indeed, an anomaly of style spines in $P$. (P.) papatasi was first described in 1979 [18]. In Albania, while surveying leishmaniasis vectors, morphological abnormalities were recorded in the three species Sergentomyia minuta Rondani, 1843, P. (P.) papatasi and Phlebotomus (Larroussius) neglectus Tonnoir, 1921, with anomalies 

Nitzulescu, 1930 (Diptera: Psychodidae), in Tunisia

in the number of spines of the styles and of the lateral lobes [19]. In Brazil, different anomalies were observed in Lutzomyia intermedia Lutz \& Neiva, 1,912 males with a specimen having supplementary sub-apical spine on each style and others showing either a third spine of the style on a protuberance or a supplementary spine in a style [20]. Still in Brazil, bilateral anomaly of the spines number on the gonostyle was found in Sciopemyia sordellii Shannon \& Del Ponte species [21] and Evandromyia evandroi species [22]. Also in Brazil, unilateral and bilateral anomalies in the paramere of Lutzomyia longipalpis Lutz \& Neiva, 1,912 males were described [23]. In Morocco, malformations of the genitalia in males of the species $P$. (P.) papatasi were widespread and largely concerned the number of spines in the lateral lobes and in the styles [24]. In Thailand, males and females of the species Chinius barbazani n. sp. were described and showed an anomaly regarding wing venation in both genders [25].

In females, frequent abnormalities concerned the number of cibarial teeth and anomalies in the shape and rarely in the number of spermathecae that were found to be associated with gynandromorphism. The non-development of one spermathecae was first reported in Sergentomyia minutia [26] and then a new case of gynandromorphism in the species Sergentomyia minuta parroti Adler \& Theodor, 1927 was reported in Algeria [27]. In Spain, gynandromorphism was found in a population of Sergentomyia minuta [28] and also in two specimens of the species $P$. (L.) perniciosus [29]. Only one report of gynandromorphism was observed in Brazil, in a specimen of Lutzomyia longipalpis and a specimen of Psychodopygus davisi Root, 1934 [30]. Also in Brazil, a female of the species Lutzomyia intermedia was found with nine cibarial horizontal teeth [20]. Our work descried for the first time in Tunisia an abnormal number of spermathecae in a $P$. (L.) longicuspis female species. To our knowledge, no anomalies have yet been described within phlebotomine sand fly populations in Tunisia. Literature review showed that only two cases of supernumerary spermathecae have been previously reported; one specimen of the $P$. (L.) perniciosus species in Portugal [31] and one specimen of the Psychodopygus carrerai Barretto, 1,946 species in Peru [32]. These authors also reported the absence of one spermathecae in one specimen of the Psychodopygus amazonensis Root, 1,934 species from Peru [32].

Morphological abnormalities are important to report in order to avoid erroneous sand fly identification and confusion in taxonomic classification which may lead to description of new species thus increasing the number of synonymous species.

The cause of malformations in sand fly genitalia remains unclear. It has been hypothesized that segregation of a recessive character under a selective pressure and injuries received by the insect during the course of its ontogeny may lead to abnormalities [33, 34]. The effect of environmental conditions on the morphological characteristics of living organisms, especially for sand flies which are known for their inability to disperse, is also well documented [35]. Indeed, the action of an insect growth inhibitor (chlorfluazuron) after its addition to the larval diet, was shown to cause morphological abnormalities of adult Lutzomyia longipalpis [36]. In another dipterous insect, Drosophila melanogaster, morphological variability of spermathecae was shown to be affected by temperature variation [37]. Recently, it has been shown that phenotypic variation in the genitalia of male $P$. (P.) papatasi is associated with environmental disturbances since they are prevalent in wild dumps and areas of application of sewage [24].

\section{Conclusions}

In this work we report for the first time an abnormal number of spermathecae in a female of the species $P$. (L.) longicuspis in Tunisia. It is important to document morphological anomalies, so as to avoid erroneous sand fly identification. The genetics and the 

Nitzulescu, 1930 (Diptera: Psychodidae), in Tunisia

possible importance of these anomalies, mostly those in the genitalia, for the copulation and survival should be evaluated.

\section{Acknowledgments}

This study received financial support from the Tropical Medicine Research Centers (P50) program (Contract No. RFA-AI-06-006), USA.

\section{References}

[1] Killick-Kendrick, R. 1990. "Phlebotomine Vectors of the Leishmaniases: A Review.” Med. Vet Entomol. 4: 1-24.

[2] WHO: Control of the leishmaniases: report of a meeting of the WHO Expert Committee on the Control of Leishmaniases. World Health Organ Tech Rep; 2010: Ser 949, Geneva.

[3] Ready, P. D. 2013. "Biology of Phlebotomine Sand Flies as Vectors of Disease Agents.” Ann. Rev. Entomol. 58: 227-50.

[4] Hambuch, T. M., Handley, S. A., Ellis, B., Chamberlin, J., Romero, S., and Regnery, R. 2004. "Population Genetic Analysis of Bartonella bacilliformis Isolates from Areas of Peru where Carrion's Disease is Endemic and Epidemic.” J. Clin. Microbiol. 42: 3675-80.

[5] Cohnstaedt, L. W., Beati, L., Caceres, A. G., Ferro, C., and Munstermann, L. E. 2011. "Phylogenetics of the Phlebotomine Sand Fly Group Verrucarum (Diptera: Psychodidae: Lutzomyia).” Am. J. Trop. Med. Hyg. 84: 913-22.

[6] Depaquit, J., Grandadam, M., Fouque, F., Andry, P., and Peyrefitte, C. 2010. "Arthropod-borne Viruses Transmitted by Phlebotomine Sand-flies in Europe: A Review.” Eurosurveillance 15: 19507.

[7] Maroli, M., Feliciangeli, M. D., Bichaud, L., Charrel, R. N., and Gradoni, L. 2013. "Phlebotomine Sand Flies and the Spreading of Leishmaniases and Other Diseases of Public Health Concern.” Med. Vet. Entomol. 27: 123-47.

[8] Rioux, J. A., Jarry, D. M., Lanotte, G., Maazoun, R., and Killick-Kendrick, R. 1984. "Ecologie des leishmanioses dans le sud de la France. Identification enzymatique de Leishmania infantum Nicolle, 1908, isolé de Phlebotomus ariasi Tonnoir, 1921 spontanément infesté en Cévennes.” Ann. Parasitol. Hum. Comp. 59: 331-3.

[9] Parrot, L., Donatien, A., and Plantureux, E. 1941. "Sur l'infection naturelle des phlébotomes par la leishmaniose générale de l’homme et du chien en Algérie.” Arch. Inst. Pasteur Alger 19: 209-18.

[10] Dedet, J. P., Addadi, K., and Belazzoug, S. 1984. "Les phlébotomes (Diptera, Psychodidae) d’Algérie. Cahiers ORSTOM.” Série Entomologie Médicale et Parasitologie
22: 99-127.

[11] Harrat, Z., Pratlong, F., Belazzoug, S., Dereure, J., Deniau, M., Rioux, J. A., Belkaid, M., and Dedet, J. P. 1996. "Leishmania infantum and Leishmania major in Algeria.” Trans. R. Soc. Trop. Med. Hyg. 90: 625-9.

[12] Berdjane-Brouk, Z., Charrel, R. N., Hamrioui, B., and Izri, A. 2012. "First Detection of Leishmania infantum DNA in Phlebotomus longicuspis Nitzulescu, 1930 from Visceral Leishmaniasis Endemic Focus in Algeria.” Parasitol. Res. 111: 419-22.

[13] Zoghlami, Z., Chouihi, E., Barhoumi, W., Dachraoui, K., Massoudi, N., Helel, K. B., Habboul, Z., Hadhri, M. H., Limam, S., Mhadhbi, M., Gharbi, M., and Zhioua, E. 2014. "Interaction between Canine and Human Visceral Leishmaniases in a Holoendemic Focus of Central Tunisia.” Acta Trop. 139: 32-8.

[14] Dantas-Torres, F., Tarallo, V., and Otranto, D. 2014. "Morphological Keys for the Identification of Italian Phlebotomine Sand Flies (Diptera: Psychodidae: Phlebotominae).” Parasit Vectors 7: 479.

[15] Léger, N., Pesson, B., Madulo-Leblond, G., and Abonnenc, E. 1983. "Sur la différenciation des femelles du sous-genre Larroussius Nitzulescu, 1931 (Diptera-Phlebotomidae) de la région Méditérranéenne.” Ann. Parasitol. Hum. Comp. 58: 611-23.

[16] Fathallah-Mili, A., Saghrouni, F., Ben Said, Z., Saadi-Ben Aoun, Y., Guizani, I., and Ben Said, M. 2012. "Retrospective Analysis of Leishmaniasis in Central Tunisia: An Update on Emerging Epidemiological Trends. In: Rodriguez-Morales A, editor. Current Topics in Tropical Medicine.” InTech. ISBN: 978-953-51-0274-8.

[17] Lewis, D. J. 1982. "A Taxonomic Review of the Genus Phlebotomus (Diptera: Psychodidae).” Bull Br Mus (Nat Hist) Entomol. 45: 121-209.

[18] Dancesco, P., and Chadli, A. 1979. "An Anomaly of Style Spines in Phlebotomus papatasi Scopoli, 1786 (Diptera, Psychodidae).” Arch Inst. Pasteur Tunis 56: 53-6.

[19] Adhami, J. 1991. "Morphological Abnormalities of Sandflies (Diptera, Psychodidae) in Albania." Parasitologia 33 (2-3): 169-73.

[20] Brisola Marcondes, C. 1999. "Anomalies of Lutzomyia intermedia (Lutz \& Neiva, 1912) (Diptera, Psychodidae, Phlebotominae).” Mem. Inst. Oswaldo Cruz. 94: 365-6.

[21] Cutolo, A. A., Zuben, C. J., and Galati, E. A. 2009. "Bilateral Anomaly in the Style of Sciopemyia sordellii (Shannon \& Del Ponte) (Diptera, Psychodidae).” Rev. Bras. Parasitol. Vet. 18: 67-9.

[22] Costa, P. L., Silva, F. J., Andrade Filho, J. D., Shaw, J. J., and Brandão Filho, S. P. 2012. "Bilateral Anomaly in Evandromyia evandroi (Diptera: Psychodidae: Phlebotominae) Captured in Vicência Municipality, Northern Rainforest Region of Pernambuco State, Brazil.” 

Nitzulescu, 1930 (Diptera: Psychodidae), in Tunisia

J. Am. Mosq. Control Assoc. 28: 128-30.

[23] Sanguinette Cde, C., Faustino, J. X., Serra e Meira, P. C., Botelho, H. A., Carvalho, G. M., Gontijo, C. M., and Filho, J. D. 2013. "Anomalies in the Sand Fly Lutzomyia longipalpis (Diptera: Psychodidae) in Brazil.” J. Am. Mosq. Control Assoc. 29: 54-8.

[24] Guernaoui, S., Ramaoui, K., Rahola, N., Barnabe, C., Sereno, D., and Boumezzough, A. 2010. "Malformations of the Genitalia in Male Phlebotomus papatasi (Scopoli) (Diptera: Psychodidae).” J. Vector Ecol. 35: 13-9.

[25] Depaquit, J., Léger, N., and Beales, P. 2006. "Chinius barbazani n. sp. from Thailand (Diptera: Psychodidae).” Parasite 13: 151-8.

[26] Rioux, J. A., Croset, H., and Maistre, M. 1974. "Case of Gynandromorphism in Sergentomyia minuta (Rondani, 1843) (Diptera-Psychodidae).” Ann Parasitol. Hum. Comp. 49: 371-2.

[27] Addadi, K., and Dedet, J. P. 1977. “A New Case of Gynandromorphism in Sergentomyia minuta parroti (Adler and Theodor, 1927) (Diptera, Psychodidae).” Arch Inst. Pasteur Alger 52: 135-8.

[28] Gállego, M., Gállego, J., Marrugat, O., Fisa, R., Portús, M., and Riera, M. C. 1991. "Gynandromorphism in a Population of Sergentomyia minuta (Rondani, 1843) in the Northeast of Spain.” Parasitologia 33: 253-60.

[29] Gállego, J., Gállego, M., Castillejo, S., Fisa, R., and Portús, M. 1994. "First Cases of Gynandromorphism in Phlebotomus perniciosus Newstead 1911 (Diptera, Psychodidae, Phlebotominae).” Parasite 1: 283-5.

[30] de Souza, M. B., Wermelinger, E. D., Ponte Cdos, S., Meira Ade, M., Machado, R. N., Vargas, P. S., and de
Carvalho, R. W. 2008. "Rare Occurrence of Gynandromorphism in Lutzomyia davisi (Diptera: Psychodidae: Phlebotominae) in Brazil.” J. Med. Entomol. 45: 166-8.

[31] de Azevedo, J. F. 1946. "Uma anomalia rara verificada numa fêmea de Phlebotomus perniciosus.” Ann. Inst. Medicina Tropical III: 183-6.

[32] Ogusuku, E., and Perez, J. E. 1994. “Abnormal Spermathecae Numbers in Peruvian Sandflies (Diptera: Psychodidae).” Acta Amazon 24: 317-20.

[33] Dedet, J. P., Addadi, K., and Belazzoug, S. 1984. "Les phlébotomes (Diptera : Psychodidae) d’Algérie.” Cah ORSTOM, sér Ent Méd Parasitol XXII: 99-127.

[34] Kassem, H., Abdel Sattar, S., Shehata, M. G., and el Sawaf, B. 1988. "Abnormalities in the sand fly Phlebotomus papatasi Scopoli (Diptera: Psychodidae) of Egypt.” J. Egypt Publ. Hlth. Assoc. 63: 209-13.

[35] Killick-Kendrick, R., Rioux, J. A., Bailly, M., Guy, M. W., Wilkes, T. J., Guy, F. M., Davidson, I., Guilvard, E., Perieres, J., and Dubois, H. 1984. "Ecology of Leishmaniasis in the South of France 20. Dispersal of Phlebotomus ariasi Tonnoir, 1921 as a factor in the spread of visceral leishmaniasis in the Cévennes.” Ann. Parasitol. Hum. Comp. 59: 555-72.

[36] Quesada, B. L., and Montoya-Lerma, J. 1994. "Laboratory Evaluation of Chlorfluazuron against Larval Phlebotomine Sand Flies (Diptera: Psychodidae).” J. Econ. Entomol. 87: 1129-32.

[37] Hadorn, E., and Graber, H. 1944. "Uber einen Drosophila, stalnnl mit veranderten spermatheken.” Rev. Suisse Zoo: 418. 